\title{
Compound-nuclear reactions with unstable nuclei: Constraining theory through innovative experimental approaches*
}

\author{
J. E. Escher ${ }^{1, a}$, A. P. Tonchev ${ }^{1}$, J. T. Burke ${ }^{1}$, P. Bedrossian ${ }^{1}$, R. J. Casperson ${ }^{1}$, N. Cooper ${ }^{2,}$, R. \\ O. Hughes ${ }^{1}$, P. Humby ${ }^{2,3}$, R. S. llieva ${ }^{3,4}$, S. Ota ${ }^{1}$, N. Pietralla ${ }^{5}$, N. D. Scielzo ${ }^{1}$, and V. Werner ${ }^{4,5}$, \\ ${ }^{1}$ Lawrence Livermore National Laboratory, Livermore, CA 94550, USA \\ ${ }^{2}$ Department of Physics, University of Richmond, Richmond, Virginia 23173, USA \\ ${ }^{3}$ Department of Physics, University of Surrey, Guildford, GU2 7XH, UK \\ ${ }^{4}$ Wright Nuclear Structure Laboratory, Yale University, New Haven, CT 06520, USA \\ ${ }^{5}$ Institut fuer Kernphysik, TU Darmstadt, Schlossgartenstrasse 9, 64289 Darmstadt, Germany
}

\begin{abstract}
Cross sections for compound-nuclear reactions involving unstable targets are important for many applications, but can often not be measured directly. Several indirect methods have recently been proposed to determine neutron capture cross sections for unstable isotopes. We consider three approaches that aim at constraining statistical calculations of capture cross sections with data obtained from the decay of the compound nucleus relevant to the desired reaction. Each method produces this compound nucleus in a different manner (via a light-ion reaction, a photon-induced reaction, or $\beta$-decay) and requires additional ingredients to yield the sought-after cross section. We give a brief outline of the approaches and employ preliminary results from recent measurements to illustrate the methods. We discuss the main advantages and challenges of each approach
\end{abstract}

\section{Introduction}

Compound-nuclear reactions play an important role in many applications. Their cross sections are required input for astrophysical models that describe stellar evolution and nucleosynthesis and for modeling processes that are relevant to generating energy. Neutron capture cross sections, for instance, are crucial for understanding the production of the elements from iron to uranium that we observe today. It is well known that nucleosynthesis of heavy elements beyond ${ }^{56} \mathrm{Fe}$ takes place primarily by neutron capture on lighter seed nuclei in the s (slow neutron capture) and $\mathrm{r}$ (rapid neutron capture) processes, with other processes contributing to the abundances of some specific isotopes. Near stability, neutron captures on s-process branch points, unstable nuclei with a lifetime long enough to allow the s process to proceed by either neutron capture or $\beta$ decay, are of particular interest. The competition between capture and decay paths depends on environmental variables, such as neutron densities, temperatures, and pressure, as well as on nuclear properties. Measured s-process abundances, therefore, yield valuable insights into the detailed conditions of the astrophysical s process. Further away from stability, one finds isotopes for which the capture cross sections affect the isotopic abundance patterns predicted

\footnotetext{
a e-mail: escher1@1lnl.gov
} 
by r-process models. While the r process is known to take place in a high-temperature, high-flux environment, neither the exact path along the nuclear chart nor the astrophysical site where it takes place is known. Capture cross sections significantly affect the isotopic abundance patterns predicted by astrophysical models and need to be known to draw conclusions about stellar evolution and nucleosynthesis. Uncovering the origin of the heavy elements is one of the most important overarching questions in nuclear science [1].

However, due to the short half-live of many of the isotopes involved in the synthesis of the heavy elements, a large number of capture cross sections cannot be measured directly. Statistical reaction calculations typically use regional systematics to determine cross sections for isotopes at and near stability. In the absence of constraining auxiliary data, cross section uncertainties can be significant. Predictions of cross sections for nuclei away from stability, moreover, rely on extrapolations and become increasingly uncertain. An order of magnitude or two difference between different statistical calculations are not unusual in the latter case.

This contribution discusses indirect methods for constraining calculations of compound-nuclear reaction cross sections with particular focus on neutron-capture reactions. Specifically, we will focus on the production of the relevant compound nuclei $(\mathrm{CN})$ via light-ion inelastic scattering and transfer reactions, photon-induced reactions, and nuclear $\beta$-decay. We will illustrate the methods with examples from recent experiments and discuss the challenges involved in extracting cross sections from the measurements.

\section{The need for indirect measurements}

\subsection{Formalism for compound-nuclear capture reactions}

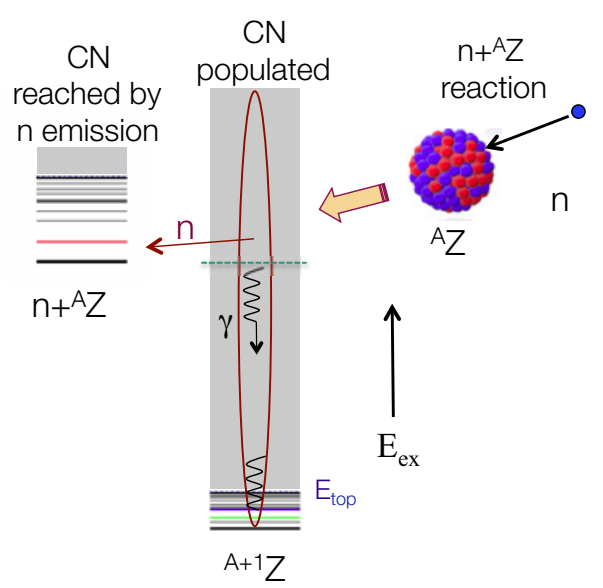

Figure 1. Schematic of a capture reaction. In a compound neutron capture process, the neutron and target nucleus ${ }^{A} \mathrm{Z}$ fuse to form the ${ }^{A+1} \mathrm{Z}$ compound nucleus, which subsequently decays via a $\gamma$-ray cascade. The capture cross section can be calculated using statistical Hauser-Feshbach theory, which requires nuclear level information, level densities, and $\gamma$-ray strength functions to properly describe the competition between the $\gamma$ channel of interest and the competing particle emission channels (Here, we show only the competition between the $\gamma$ and neutron channels.) 
The formalism appropriate for describing compound-nucleus reactions is the statistical HauserFeshbach theory [2-4]. The average cross section per unit energy in the outgoing channel for reactions proceeding to an energy region in the final nucleus described by a level density is given by:

$$
\frac{d \sigma_{\alpha \chi}^{H F}\left(E_{a}\right)}{d E_{\chi}}=\pi \lambda_{\alpha}^{2} \sum_{J \pi} \omega_{\alpha}^{J} \sum_{\ell s \ell^{\prime} s^{\prime} I^{\prime}} \frac{T_{\alpha \ell s}^{J} T_{\chi \ell^{\prime} s^{\prime}}^{J} \rho_{I^{\prime}}\left(U^{\prime}\right) W_{\alpha \chi}(J)}{\sum_{\chi^{\prime \prime} \ell^{\prime \prime} s^{\prime \prime}}^{\prime} T_{\chi^{\prime \prime} \ell^{\prime \prime} s^{\prime \prime}}^{J}+\sum_{\chi^{\prime \prime} \ell^{\prime \prime} s^{\prime \prime} I^{\prime \prime}} \int T_{\chi^{\prime \prime} \ell^{\prime \prime} s^{\prime \prime}}^{J}\left(E_{\chi^{\prime \prime}}\right) \rho_{I^{\prime \prime}}\left(U^{\prime \prime}\right) d E_{\chi^{\prime \prime}}} .
$$

Here $\alpha$ denotes the entrance channel $a+A$ and $\chi$ represents the relevant exit channel $c+C, E_{a}$ is the kinetic energy of the projectile, and $\lambda_{\alpha}$ is the reduced wavelength in the incident channel. The spin of the incident particle is $i$, the target spin is $I$, the channel spin is $\vec{s}=\vec{\imath}+\vec{I}$, and the compound-nucleus angular momentum and parity are $J \pi$. The statistical-weight factor $\omega_{\alpha}^{J}$ is $(2 J+1) /[(2 i+1)(2 I+1)]$. Similarly, the spin of the outgoing particle is $i^{\prime}$, the spin of the residual nucleus is $I^{\prime}$, and the channel spin for $\chi$ is $\vec{s}=\vec{\imath}+\vec{I}^{\prime}$. The quantities $\ell$ and $\ell^{\prime}$ are the relative orbital angular momentum in the entrance and exit channels, respectively. The transmission coefficients are written as $T_{\alpha l s}^{J}$ and $\rho_{I^{\prime}}\left(U^{\prime}\right)$ denotes the density of levels of spin $I^{\prime}$ at excitation energy $U^{\prime}$ in the residual nucleus. All energetically possible final channels $\chi^{\prime \prime}$ have to be taken into account, thus the denominator includes contributions from decays to discrete levels in the residual nuclei (given by the first sum in the denominator, $\Sigma^{\prime}$ ) as well as contributions from decays to regions described by a level density in the residual nuclei (given by the second sum in the denominator which involves an energy integral of transmission coefficients and level densities in the residual nuclei). Width fluctuation corrections $W_{\alpha \chi}$ are included in order to account for correlations between the incident and outgoing reaction channels [5,6]. In writing Eq. 1, we have suppressed the parity quantum number except for that of the compound nucleus. In fact, the level density depends in principle on parity and all sums over quantum numbers must respect parity conservation.

For radiative neutron capture $\left(\alpha=\mathrm{n}+{ }^{A} \mathrm{Z}\right.$ and $\left.\chi=\gamma+{ }^{A+1} \mathrm{Z}\right)$, we usually need only the integral over the energy spectrum of primary $\gamma$ rays emitted from the compound nucleus. (To determine cross sections for particular $\gamma$ transitions or for the production of isomers, additional details of the $\gamma$-cascade need to be accounted for.) We integrate over all energies $E_{\chi}$ of the final-state channel and, in a first approximation, neglect the width fluctuation correlations. The primary effect of the correlations is an enhancement of the elastic scattering cross section. This allows us to write the cross section for the desired reaction as:

$$
\sigma_{\alpha \chi}\left(E_{n}\right)=\sum_{J, \pi} \sigma_{\alpha}^{C N}\left(E_{e x}, J, \pi\right) G_{\chi}^{C N}\left(E_{e x}, J, \pi\right)
$$

where $\sigma^{C N}\left(E_{e x}, J, \pi\right)=\sigma\left(\mathrm{n}+{ }^{A} Z \rightarrow{ }^{A+1} Z^{*}\right)$ denotes the cross section for forming the compound nucleus at excitation energy $E_{e x}$ with angular-momentum and parity quantum numbers $J \pi$ and $G_{\chi}^{C N}\left(E_{e x}, J, \pi\right)$ is the branching ratio for the decay of this compound state into the desired exit channel $\chi$. It contains transmission coefficients for the competing exit channels as well as the associated level densities and information on discrete levels. The kinetic energy $E_{n}$ of the neutron is related to the excitation energy of the compound nucleus, $E_{e x}$, via $E_{n}=\frac{A+1}{A}\left(E_{e x}-S_{n}\right)$, where $S_{n}$ is the energy required for separating a neutron from the compound nucleus ${ }^{A+1} Z$. This factored form embodies the essential assumptions of the Hauser-Feshbach model, that formation and decay of the compound nucleus are independent processes, and that the total spin and parity of the compound system must be conserved.

\subsection{Ingredients for calculating capture cross sections}

Ingredients required to carry out Hauser-Feshbach cross-section calculations include nuclear binding energies, spins and parities of both ground and excited nuclear states, $\gamma$-branching ratios for these 
states, nuclear level densities and transmission coefficients for particles, photons, and fission. Much effort has been devoted to develop the requisite models and codes, and to formulate parameter recommendations [7]. Auxiliary experiments provide important constraints for these models and their parameters. For instance, neutron resonance measurements provide average level spacings and thus constraints for the level densities near the neutron separation energy. Average radiative widths provide information on the product of the level density and the $\gamma$-ray strength function, which is relevant to capture cross section calculations.

For neutron capture reactions involving nuclei near the valley of stability, the formation of the compound nucleus is reasonably well described, while a reliable description of the decay is more challenging, since the competition between all possible decay channels has to be properly accounted for in the calculated decay probabilities. Specifically, the $\gamma$-ray strength function for the $\mathrm{CN}^{A+1} Z$ is needed and the level densities in both the target $\left({ }^{A} Z\right)$ and compound nuclei are required, as well as information on the low-lying discrete states (energies, spins and parities) in the target nucleus.

For reactions involving unstable targets, the requisite neutron resonance spacings and average radiative capture widths are not available and the decay probabilities $G_{\chi}^{C N}\left(E_{e x}, J, \pi\right)$ become the primary source of uncertainty in calculations of capture cross sections. Evaluations typically rely on regional systematics in this case and a factor two (or more) difference between evaluated cross sections is not uncommon; for reactions involving nuclei far from stability the uncertainties increase to more than an order of magnitude. The indirect approaches discussed in this contribution aim at improving this situation by providing constraints for these $G_{\chi}^{C N}\left(E_{e x}, J, \pi\right)$.

\section{$3 \mathrm{CN}$ production via inelastic scattering and transfer reactions}

\subsection{Method and illustrative example}

In an approach that has become known as the 'surrogate method' [8], the compound nucleus ${ }^{A+1} Z^{*}$ is produced by an inelastic scattering or transfer reaction $d+D \rightarrow b+{ }^{A+1} Z^{*}$, and the desired decay channel $\chi\left({ }^{A+1} Z^{*} \rightarrow c+C\right.$ ) is observed in coincidence with the outgoing particle $b$ (see left panel of Fig. 2). The probability for forming ${ }^{A+1} Z^{*}$ in the surrogate reaction (with specific values for $E_{e x}, J, \pi$ ) is $F_{\delta}^{C N}\left(E_{e x}, J, \pi, \theta_{b}\right)$, where $\delta$ refers to the entrance channel reaction $D(d, b)$ and $\theta_{b}$ is the angle of the outgoing direct-reaction particle $b$ relative to the beam axis. The quantity

$$
P_{\delta \chi}\left(E_{e x}, \theta_{b}\right)=\sum_{J, \pi} F_{\delta}^{C N}\left(E_{e x}, J, \pi, \theta_{b}\right) G_{\chi}^{C N}\left(E_{e x}, J, \pi\right),
$$

which gives the probability that the compound nucleus ${ }^{A+1} Z^{*}$ was formed with energy $E_{\text {ex }}$ and decayed into channel $\chi=\gamma+{ }^{A+1} Z$, can be obtained experimentally, by measuring $N_{\delta}$, the total number of surrogate events, and $N_{\delta \chi}$, the number of coincidences between the direct-reaction particle and the observable that identifies the relevant exit channel ${ }^{1}: P_{\delta \chi}^{e x p}\left(E_{e x}, \theta_{b}\right)=N_{\delta \chi}\left(E_{e x}, \theta_{b}\right) / N_{\delta}\left(E_{e x}, \theta_{b}\right) \epsilon_{\delta}\left(E_{e x}\right)$. Here, $\epsilon_{\delta}\left(E_{e x}\right)$ denotes the efficiency for detecting the exit-channel $\chi$ (when in coincidence with the outgoing direct-reaction particle $b$ ). For example, a discrete $\gamma$-ray transition involving low-lying states in the decaying $\mathrm{CN}^{A+1} Z^{*}$ can be used to identify the capture channel. In that case, $\epsilon_{\delta}\left(E_{e x}\right)$ is the efficiency for detecting a $\gamma$-ray of that energy experimentally (preferably in a high-resolution Germanium detector).

To illustrate the idea, we discuss preliminary results from an experiment in the $\mathrm{Zr}$ region. The decay of compound nuclei in this region is known to be particularly sensitive to the spins and parities

\footnotetext{
${ }^{1}$ The angular dependence of $P_{\delta \chi}^{e x p}\left(E_{e x}, \theta_{b}\right)$ arises from the fact that the population $F_{\delta}^{C N}\left(E_{e x}, J, \pi, \theta_{b}\right)$ of the compound nucleus depends on the angular-momentum transferred in the surrogate reaction, and hence on $\theta_{b}$.
} 
populated via a surrogate reaction [9-13]. Figure 2 gives a schematic representation of the surrogate ${ }^{92} \mathrm{Zr}\left(\mathrm{p}\right.$,d) reaction, which was recently used to produce the $\mathrm{CN}{ }^{91} \mathrm{Zr}$ at a range of excitation energies. This case is relevant to the ${ }^{91} \operatorname{Zr}(\mathrm{n}, \gamma)$ reaction, for which directly-measured cross-section data exists. The experiment was carried out at the Texas A\&M University Cyclotron Institute, where the K150 Cyclotron was employed to provide a $28.5-\mathrm{MeV}$ proton beam. Particle- $\gamma$ coincidence data was collected using the STARLiTeR array, a combination of a silicon telescope array and five HPGe clover detectors (see, e.g., Ref. [13]). The outgoing deuteron was detected at angles around 30-60 deg relative to the beam direction, and $\gamma$-rays associated with transitions between low-lying states $\left(E_{n}<2 \mathrm{MeV}\right)$ were measured. The data in the right panel of Figure 2 gives the coincidence probability for the $1466 \mathrm{keV}$ transition (from the $5 / 2^{+}$state at $1.466 \mathrm{MeV}$ to the $5 / 2^{+}$ground state of ${ }^{91} \mathrm{Zr}$ ) as a function of the excitation energy $E_{e x}$ in the ${ }^{91} \mathrm{Zr}$ nucleus. $E_{e x}$ was reconstructed from the measured energy and angle of the outgoing deuteron. When the $\mathrm{CN}^{91} \mathrm{Zr}$ is populated below the neutron threshold $\left(S_{n}=7.15\right.$ $\mathrm{MeV}$ ), less than $10 \%$ of the decays proceed through this transition. The remaining $\gamma$-decay by-passes this transition, a fact that reflects the rich structure of this odd-A nucleus. As the excitation energy is increased beyond $S_{n}$, neutron emission begins to compete and the $\gamma$-probability drops quickly. The solid, dash-dotted, and dashed curves are the results of calculations that combine predictions for the weights $F_{\delta}^{C N}\left(E_{e x}, J, \pi, \theta_{b}\right)$ in Eq. 3 with a Hauser-Feshbach-type decay model for ${ }^{91} \mathrm{Zr}$.

The distribution $F_{\delta}^{C N}\left(E_{e x}, J, \pi, \theta_{b}\right)$, which may be very different from the CN spin-parity populations following the absorption of a neutron in the desired reaction, has to be determined theoretically, so that the branching ratios $G_{\chi}^{C N}\left(E_{e x}, J, \pi\right)$ can be extracted from the measurements. In practice, the decay of the $\mathrm{CN}$ is modeled using a Hauser-Feshbach-type decay model and the $G_{\chi}^{C N}\left(E_{e x}, J, \pi\right)$ are obtained by adjusting parameters in the model to reproduce the measured probabilities $P_{\delta \chi}\left(E_{e x}, \theta_{b}\right)$. Subsequently, the sought-after cross section can be obtained by combining the calculated cross section $\sigma_{\alpha}^{C N}\left(E_{e x}, J, \pi\right)$ for the formation of ${ }^{A+1} Z^{*}$ (from $n+{ }^{A} Z$ ) with the extracted decay probabilities $G_{\chi}^{C N}\left(E_{e x}, J, \pi\right)$ for this state, see Eq. 2 . At this point, the width fluctuation correlations can be included using standard correction factors [5, 6, 14].

The present case involves stable nuclei for which information on levels, level densities, and $\gamma$-ray strength functions is available, so the $\gamma$-cascade can be modeled and tests of the theory predicting the CN population $F_{\delta}^{C N}\left(E_{e x}, J, \pi, \theta_{b}\right)$ can be carried out. The ${ }^{92} \mathrm{Zr}(\mathrm{p}, \mathrm{d})$ reaction involves the pickup of neutrons from inner shells of the ${ }^{92} \mathrm{Zr}$ nucleus. Neutron hole states $2 \mathrm{p}_{1 / 2}, 2 \mathrm{p}_{3 / 2}, 1 \mathrm{f}_{5 / 2}, 1 \mathrm{f}_{7 / 2}$, etc., are involved in the production of ${ }^{91} \mathrm{Zr}$ near $S_{n}$. Their location and fragmentation as a function of ${ }^{91} \mathrm{Zr}$ excitation energy was obtained using the dispersive optical model approach of Mahaux and Sartor [15]. Cross sections for the one-neutron pickup process were calculated in the 1-step distorted-wave Born approximation and the relative weights of the final spins were determined as a function of $E_{e x}\left({ }^{91} \mathrm{Zr}\right)$. The combination of these with the Hauser-Feshbach-type decay model results in predicted surrogate probabilities $P_{\delta x}^{e x p}\left(E_{e x}, \theta_{b}\right)$ shown in the right panel of Fig. 2. We see that the probability calculated in the 1-step DWBA (solid red curve) does not agree well with the data. Contributions from two-step processes such as (p,p',d) and (p,d',d), in which the initial ${ }^{92} \mathrm{Zr}$ and the final ${ }^{91} \mathrm{Zr}$ are inelastically excited, are found to play an important role, as the dash-dotted and dashed curves show. Similar results are found for other $\gamma$-ray transitions in this nucleus (not shown). It becomes clear that in order to reproduce the surrogate data, structure information involving highly-excited regions of the ${ }^{91} \mathrm{Zr}$ nucleus is needed, as well as a detailed treatment of the surrogate reaction mechanism. The findings also underscore the value of benchmark experiments.

\subsection{Advantages and challenges of $\mathrm{CN}$ production via light-ion reactions}

An advantage of using inelastic scattering and transfer reactions to produce the $\mathrm{CN}$ of interest lies in the variety of projectile-target combinations that can be utilized. That makes it possible to reach a 

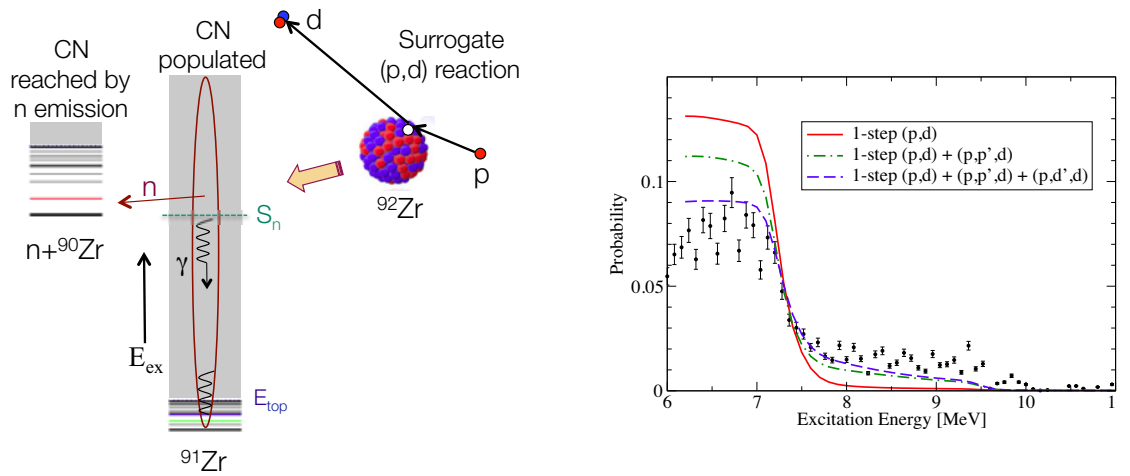

Figure 2. Strategy for obtaining cross section constraints from a (surrogate) light-ion inelastic scattering or transfer reaction. Left panel: Schematic representation of the surrogate reaction mechanism. The basic idea of the approach is to replace the first step of the desired reaction, $a+A$, by an alternative reaction, $d+D \rightarrow b+B^{*}$, that is experimentally easier to access yet populates the same compound nucleus. The subsequent decay of the compound nucleus into the relevant channel, $c+C$, can then be measured and used to extract the desired cross section. Here the desired reaction is ${ }^{90} \mathrm{Zr}(\mathrm{n}, \gamma)^{91} \mathrm{Zr}$. The $\mathrm{CN}^{91} \mathrm{Zr}$ is produced via the ${ }^{92} \mathrm{Zr}(\mathrm{p}, \mathrm{d})^{91} \mathrm{Zr}$ one-nucleon pickup reaction. Neutron evaporation and $\gamma$ emission compete in the decay of the $\mathrm{CN}$. Characteristic $\gamma$-ray transitions in ${ }^{91} \mathrm{Zr}$ are measured in coincidence with the outgoing light ion. Right panel: Measured coincidence probability in the decay of ${ }^{91} \mathrm{Zr}$. The probability of observing the $1466 \mathrm{keV}$ transition in coincidence with the outgoing deuteron is given as function of $E_{e x}$ (black data points with error bars). The curves show predicted coincidence probabilities based on one-step and two-step DWBA calculations. These results are preliminary.

large number of isotopes, in particular when the technique can be combined with radioactive beams in inverse-kinematics experiments [16]. In some cases the $\mathrm{CN}$ can be produced by multiple reactions which result in different spin-parity populations [12, 13], allowing for comparisons of cross sections obtained from different measurements and analyses. In addition, measurements of the outgoing light ion enable us to scan through a range of excitation energies in the $\mathrm{CN}$ and to obtain information both below and above the separation energy. This can be employed to probe different aspects of the $\mathrm{CN}$ decay.

To extract accurate neutron capture cross sections from surrogate data, we have to quantitatively account for the fact that the weights $F_{\delta}^{C N}\left(E_{\mathrm{ex}}, J, \pi, \theta_{b}\right)$, by which the decay probabilities $G_{\chi}^{C N}\left(E_{e x}, J, \pi\right)$ are multiplied in Eq. 3, are different from the relative formation cross sections $f_{\alpha}^{C N}\left(E_{e x}, J, \pi\right) \equiv$ $\sigma_{\alpha}^{C N}\left(E_{e x}, J, \pi\right) / \sum_{J^{\prime} \pi^{\prime}} \sigma_{\alpha}^{C N}\left(E_{e x}, J^{\prime}, \pi^{\prime}\right)$ of the desired reaction, see Eq. 2. The decay probabilities $G_{\chi}^{C N}\left(E_{e x}, J, \pi\right)$ depend on the spins and parities of the decaying nucleus and that dependence is particularly strong in the $\mathrm{Zr}$ region, where the level density is low and neutron emission to discrete levels competes with $\gamma$ emission $[9,10]$. This is different from surrogate applications to (n,f) reactions. In the fission case the $G_{\chi=\text { fission }}^{C N}\left(E_{e x}, J, \pi\right)$ are much less sensitive to spin and parity and approximations can be employed which allow for analysis methods that do not require the knowledge of the $\mathrm{CN}$ spin-parity distributions [17-20].

Predicting these weights requires a framework for calculating cross sections of different reactions (stripping, pick-up, charge exchange, and inelastic scattering) to continuum states, for a variety of projectiles ( $p, d, t, \alpha$, etc.) and targets (spherical, deformed, and transitional). The ${ }^{92} \mathrm{Zr}$ example discussed showed that pickup reactions require information on the structure of the $\mathrm{CN}$ up to very high 
excitation energies ( $\sim 10 \mathrm{MeV}$ for capture). This can be a challenge, as both (quasi) single-particle and collective degrees of freedom can be relevant. Similar considerations apply to inelastic scattering, which involves (quasi) particle-hole excitations, and stripping reactions, which involve resonance states. The example also shows that details of the reaction mechanism need to be considered. Multistep contributions can play a role and breakup (primarily for stripping reactions) needs to be accounted for.

\section{$4 \mathrm{CN}$ production via photon-induced reactions}

\subsection{Method and illustrative example}

Photon-induced reactions provide an additional mechanism for producing highly-excited nuclei, the decay of which is relevant to unknown capture cross sections. Inelastic scattering and photo-neutron reactions can be used to populate a nucleus of interest across a wide range of excitation energies, up to and beyond the neutron separation energy. Bremsstrahlung photons as well as quasi-monoenergetic $\gamma$-ray beams have been used for this purpose [21-29]. In a few experiments, neutrons emitted from the $\mathrm{CN}$ were detected and the $(\gamma, \mathrm{n})$ cross sections were determined. Hauser-Feshbach calculations of these photo-neutron reactions were carried out and comparisons with the data were utilized to determine the most appropriate combination of level densities and $\gamma$-ray strength functions [28, 30]. These were subsequently employed to calculate the $(\mathrm{n}, \gamma)$ cross section of interest.

Alternatively, it is possible to measure the $\gamma$-ray strength function, an important ingredient for neutron-capture calculations, in photon-scattering experiments [22-24]. This approach makes it possible to determine the strength function below the particle emission threshold directly, without the need to model the reaction mechanism. As electric dipole transitions dominate, this approach provides access to the $\mathrm{E} 1$ strength function. Some setups also allow one to distinguish between different multipolarities [27, 29].

The idea of the approach is illustrated in Figure 3 for the $\gamma+{ }^{76} \mathrm{Ge}$ case. Nuclear resonance fluorescence (NRF) measurements of the nuclear dipole response of ${ }^{76} \mathrm{Ge}$ were recently carried out at the $\mathrm{HI} \gamma \mathrm{S}$ facility at TUNL at Duke University and at the DHIPS facility at TU Darmstadt, Germany: Werner and collaborators et al. [31, 32] combined results from a bremsstrahlung experiment with an experiment using quasi-monoenergetic, linearly polarized photons to measure spins and parities of 130 excited levels in ${ }^{76} \mathrm{Ge}$ and to determine the photoabsorption cross section for this nucleus in the energy range between $E_{\gamma}=4 \mathrm{MeV}$ and $E_{\gamma}=9 \mathrm{MeV}$. The photoabsorption cross section is directly related to the $\gamma$-ray strength function $f\left(E_{\gamma}\right)$ of interest, $\sigma_{a b s}=3(\pi h c)^{2} E_{\gamma} f\left(E_{\gamma}\right)$. Here the assumption is made that the upward and downward strength functions are identical. We selected this example because it involves the same $\mathrm{CN}$ that has recently been produced via $\beta$-decay of ${ }^{76} \mathrm{Ga}$ and a comparison of the strength functions obtained from these very different experiments, using very different analysis techniques, can be carried out. The results will be discussed below.

\subsection{Advantages and challenges of $\gamma$-induced reactions}

The primary advantage of obtaining constraints for $(\mathrm{n}, \gamma)$ calculations from photon-induced reactions lies in the fact that this approach provides access to the $\gamma$-ray strength function in a model-independent manner (although it relies on a few assumptions, such as the validity of the Brink-Axel hypothesis). Well-established experimental techniques can be used to carry out the measurements. Recent improvements to the technique, such as the combination of an intense mono-energetic photon beam at the $\mathrm{HI} \gamma \mathrm{S}$ facility with $\gamma-\gamma$ coincidence spectroscopy of the subsequent decays makes it possible to 

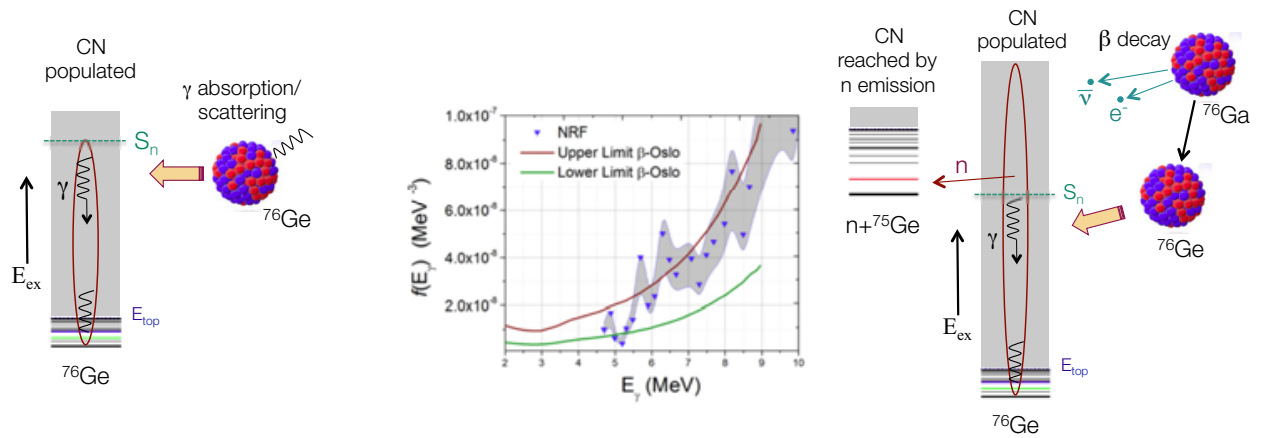

Figure 3. Strategies for obtaining cross section constraints from $\gamma$-induced reactions and nuclear $\beta$ decay. Left panel: Schematic representation of the CN production via $\gamma$-induced reactions. Photons can be elastically or inelastically scattered or absorbed. Populating states below particle thresholds gives information on the $\gamma$-ray strength function, while producing the $\mathrm{CN}$ above $S_{n}$ allows for a study of the competition between neutron and $\gamma$ emission. Right panel: Schematic representation of the $\mathrm{CN}$-production mechanism via $\beta$-decay. Center panel: Comparison of $\gamma$-ray strength functions extracted from from the photoabsorption cross section measured via nuclear resonance fluorescence experiments ( $\gamma$ absorption and emission) and a $\beta$-Oslo experiment, respectively. The former gives a more direct (model-independent) measurement of this quantity and thus provides a useful test of the latter method. The NRF results are preliminary.

study the detailed structure of the strength function in more detail than previously possible, in an energy regime that is important for neutron-capture calculations.

The fact that the spins populated in photon-induced reactions are determined by the ground state spin of the ${ }^{A+1} \mathrm{Z}$ nucleus used in the experiment can be an advantage when photo-neutron data is used to constrain Hauser-Feshbach calculations. If both the target nucleus ${ }^{A} \mathrm{Z}$ and the compound nucleus ${ }^{A+1} \mathrm{Z}$ of the desired ${ }^{A} \mathrm{Z}(\mathrm{n}, \gamma){ }^{A+1} \mathrm{Z}$ have similar spins, it can be expected that both reactions populate states of similar spins in the ${ }^{A+1} Z$ system, and thus both probe the competition of $\gamma$ decay and neutron emission from states that are similar to each other. For cases with very different spins for the ${ }^{A} Z$ and ${ }^{A+1} \mathrm{Z}$ ground state, however, we can expect a spin-mismatch between the compound nuclei created in the different reactions. This is similar to the situation discussed above for light-ion reactions, although the effect of the electromagnetic interaction is well known and the spins and parities are accounted for in a straight-forward manner in the Hauser-Feshbach calculations.

The main drawback of using photon-induced reactions is the fact that it is limited to cases where the $\mathrm{CN}$ of interest has a stable ground state. It is therefore not possible to apply this strategy to nuclei that are far from stability. A limitation of solely considering the energy regime below the neutron threshold is that only the $\gamma$-ray strength function is obtained, while additional information is required to constrain the level densities of the nuclei involved.

\section{$5 \mathrm{CN}$ production via $\beta$-decay}

\subsection{Method and illustrative example}

For reactions involving compound nuclei on the neutron-rich side of the valley of stability, a third indirect approach can be considered: Nuclear $\beta$-decay from an unstable precursor nucleus ${ }^{A+1}(\mathrm{Z}-1)$ can be used to produce the isotope of interest ${ }^{A+1} Z$. This is schematically shown in the right panel 
of Figure 3. The $\beta$-decay $\mathrm{Q}$ value determines the excitation energies that can be reached in the ${ }^{A+1} \mathrm{Z}$ nucleus. Near stability, the available energy range may not reach up to the neutron separation energy. E.g., for the ${ }^{76} \mathrm{Ga} \rightarrow{ }^{76} \mathrm{Ge}$ example shown, states up to $E_{e x}=7.0 \mathrm{MeV}$ can be populated, which is below the neutron threshold $\left(S_{n}=9.4 \mathrm{MeV}\right)$. With increasing neutron excess, the neutron binding energy decreases, the Q-value window increases, and consequently bound as well as neutron unbound states can be populated via $\beta$-decay.

Since allowed Gamov-Teller decay is the dominant mode, the spins populated in the ${ }^{A+1} \mathrm{Z}$ nucleus lie within a narrow band $\left(J^{C N}=J^{\text {pre }}-1, J^{\text {pre }}, J^{\text {pre }}+1\right.$, where $J^{\text {pre }}$ is the ground state spin of the decaying precursor). When states above $S_{n}$ are reached by $\beta$-decay, the subsequent competition between neutron and $\gamma$ emission can be studied.

The ${ }^{76} \mathrm{Ga} \rightarrow{ }^{76} \mathrm{Ge}$ case was investigated recently by a group at the National Superconducting Cyclotron Laboratory at Michigan State University (NSCL/MSU) [33]. The experiment focused on measuring $\beta$-delayed $\gamma$ emission with a total absorption $\gamma$ spectrometer (TAGS). For $\gamma$ emission in coincidence with $\beta$ particles, the authors determined the matrix $P\left(E_{\gamma}, E_{e x}\right)$, which gives the relative probabilities of primary transitions with energy $E_{\gamma}$ in ${ }^{76} \mathrm{Ge}$ originating from energy bins at excitation energies $E_{e x}$. This matrix was obtained up to about $7 \mathrm{MeV}$ and subsequently analyzed using the Oslo method [34-36].

The Oslo method was previously applied to $\gamma$-spectra measured in coincidence with chargedparticle reactions. The analysis is aimed at extracting both level density and $\gamma$-ray strength function of a decaying nucleus from an experimental primary $\gamma$-ray matrix $P\left(E_{\gamma}, E_{e x}\right)$. The analysis involves a series of steps in which the detector response function is accounted for, the primary $\gamma$-rays are isolated and the functional forms of the level density and $\gamma$-ray strength function are extracted [36]. Auxiliary data is required to determine the final physical solutions of these sought-after quantities. Typically, the level density is fixed by the requirement that it reproduces the cumulative number of levels at low energies and the neutron resonance spacing at the neutron separation energy, and the strength function is fixed by utilizing the average radiative width $\left\langle\Gamma_{\gamma}\right\rangle$ at $S_{n}$. For the ${ }^{76}$ Ge nucleus this information is not available and the authors used estimates based on microscopic calculations and systematics. The resulting level density and strength function were then used in a Hauser-Feshbach calculation of the ${ }^{75} \mathrm{Ge}(\mathrm{n}, \gamma)^{76} \mathrm{Ge}$ cross section. The cross section was found to be in agreement with standard Talys calculations. Unfortunately, no directly-measured data is available to benchmark the extracted capture cross section.

To better assess the promise of the $\beta$-Oslo approach, we thus compared the $\gamma$-ray strength function obtained in this approach with a more direct measurement of this quantity using the NRF approach discussed in the previous section. We find that, even though the NRF experiment yields a $\gamma$-ray strength function that has more structure and is slightly larger than the result from the $\beta$-Oslo method, both approaches give similar results (center panel of Figure 3). This additional, more direct, test of the $\beta$-Oslo method is encouraging. It would be useful to also carry out an independent test for the extracted level density.

\subsection{Advantages and challenges of the $\beta$-decay method}

A strong advantage of employing the $\beta$-decay approach is the fact that one can produce neutronrich isotopes far from stability, with production rates that are potentially much higher than those obtained via light-ion inverse-kinematics reactions. The $\mathrm{Q}$ values for these nuclei make it possible to populate states both below and above the neutron separation energy. Since Gamov-Teller decay dominates, the spins populated in the intermediate nucleus lie in a narrow range and are determined by the ground state spin of the decaying precursor. Knowledge of the states populated is needed in 
order to infer information on the relative probabilities of the neutron and $\gamma$ decay channels and to use that to constrain cross section calculations for capture reactions ${ }^{2}$.

The Oslo analysis method seems to be less sensitive to the spin distribution of the $\gamma$-decaying nucleus than a $\gamma$-decay analysis that makes use of individual $\gamma$ transitions. On the other hand, it has the drawback that additional, auxiliary information is required to normalize the level densities and $\gamma$-ray strength functions extracted. The data that is typically used to allow for this normalization, will most likely not be available for nuclei away from the valley of stability and alternative methods for obtaining the normalization factors need to be found.

\section{Conclusions}

Neutron-capture reactions on unstable isotopes are of interest to astrophysics and other applications. Indirect measurements play an important role in constraining calculations of the requisite cross sections. Several innovative methods for obtaining experimental constraints for the calculations are being explored. We have considered three approaches that produce the compound nuclei of interest via light-ion inelastic scattering and transfer reactions, photon-induced reactions, and nuclear $\beta$-decay, respectively. The constraints are obtained from measurements of the subsequent compound-nuclear decay. Each method has its unique strengths, as well as experimental and theoretical challenges that have to be overcome in order to provide reliable capture cross sections for nucleosynthesis modeling and other applications. Having several methods at one's disposal is important in order to validate the results obtained and to cover the reactions of interest.

Current investigations focus mostly on isotopes close to stability, where benchmarking is facilitated by the availability of auxiliary information and, in some cases, directly-measured cross sections. The longer-term goal is to develop methods that can be used for a wider range of reactions. Radioactive beams are now available at multiple facilities, such as Argonne National Laboratory (ANL) and the National Superconducting Cyclotron Laboratory at Michigan State University (NSCL/MSU). FRIB, which is under construction at MSU, will provide more intense beams of isotopes far from stability. The availability of these exotic beams make it possible to produce important isotopes using inelastic scattering and transfer reactions in inverse kinematics, as well as via $\beta$-decay, and to study of the subsequent competition between neutron and $\gamma$-ray emission.

\section{Acknowledgments}

This work was performed under the auspices of the U.S. Department of Energy by Lawrence Livermore National Laboratory under contract DE-AC52-07NA27344, with partial support through LLNL's LDRD (15-ERD-069) and ASC/PEM programs.

\section{References}

[1] Nuclear Science Advisory Committee, Reaching for the Horizon: The 2015 Long Range Plan for Nuclear Science (2015)

[2] W. Hauser, H. Feshbach, Phys. Rev. 87, 366 (1952)

[3] E. Gadioli, P.E. Hodgson, Pre-Equilibrium Nuclear Reactions (Clarendon Press, Oxford, 1992)

[4] P. Fröbrich, R. Lipperheide, Theory of Nuclear Reactions (Clarendon Press, Oxford, 1996)

\footnotetext{
${ }^{2}$ An attempt to determine this competition by combining $\beta$-delayed $\gamma$ data with $\beta$-delayed neutron data was recently published for the ${ }^{87} \mathrm{Br} \rightarrow{ }^{87} \mathrm{Kr},{ }^{88} \mathrm{Br} \rightarrow{ }^{88} \mathrm{Kr}$, and ${ }^{94} \mathrm{Rb} \rightarrow{ }^{94} \mathrm{Zr}$ cases [37]. The results of that experiment implied some unusual behavior of the strength function and need to be understood better.
} 
[5] P.A. Moldauer, Phys. Rev. 123, 968 (1961)

[6] S. Hilaire, C. Lagrange, A.J. Koning, Annals of Physics 306, 209 (2003)

[7] R. Capote, M. Herman, P. Oblozinský, P. Young, S. Goriely, T. Belgya, A. Ignatyuk, A. Koning, S. Hilaire, V. Plujko et al., Nuclear Data Sheets 110, 3107 (2009)

[8] J.E. Escher, J.T. Burke, F.S. Dietrich, N.D. Scielzo, I.J. Thompson, W. Younes, Rev. Mod. Phys. 84, 353 (2012)

[9] C. Forssén, F. Dietrich, J. Escher, R. Hoffman, K. Kelley, Phys. Rev. C 75, 055807 (2007)

[10] J.E. Escher, F.S. Dietrich, Phys. Rev. C 81, 024612 (2010)

[11] N.D. Scielzo, J.E. Escher, J.M. Allmond, M.S. Basunia, C.W. Beausang, L.A. Bernstein, D.L. Bleuel, J.T. Burke, R.M. Clark, F.S. Dietrich et al., Phys. Rev. C 81, 034608 (2010)

[12] S. Ota, J. Burke, R. Casperson, J. Escher, R. Hughes, J. Ressler, N. Scielzo, I. Thompson, R. Austin, E. McCleskey et al., EPJ Web of Conferences 93, 02001 (2015)

[13] S. Ota, J.T. Burke, R.J. Casperson, J.E. Escher, R.O. Hughes, J.J. Ressler, N.D. Scielzo, I.J. Thompson, R.A.E. Austin, B. Abromeit et al., Phys. Rev. C 92, 054603 (2015)

[14] T. Kawano, P. Talou, H.A. Weidenmüller, Phys. Rev. C 92, 044617 (2015)

[15] C. Mahaux, R. Sartor, Adv. Nucl. Phys. 20, 1 (1991)

[16] J. Cizewski, R. Hatarik, K. Jones, S. Pain, J. Thomas, M. Johnson, D. Bardayan, J. Blackmon, M. Smith, R. Kozub, Nuclear Instruments and Methods in Physics Research Section B: Beam Interactions with Materials and Atoms 261, 938 (2007)

[17] J.E. Escher, F.S. Dietrich, Phys. Rev. C 74, 054601 (2006)

[18] J.T. Burke, L.A. Bernstein, J. Escher, L. Ahle, J.A. Church, F.S. Dietrich, K. Moody, E.B. Norman, L. Phair, P. Fallon et al., Phys. Rev. C 73, 054604 (2006)

[19] G. Kessedjian, B. Jurado, M. Aiche, G. Barreau, A. Bidaud, S. Czajkowski, D. Dassié, B. Haas, L. Mathieu, L. Audouin et al., Physics Letters B 692, 297 (2010)

[20] J.J. Ressler, J.T. Burke, J.E. Escher, C.T. Angell, M.S. Basunia, C.W. Beausang, L.A. Bernstein, D.L. Bleuel, R.J. Casperson, B.L. Goldblum et al., Phys. Rev. C 83, 054610 (2011)

[21] R. Schwengner, R. Beyer, F. Dönau, E. Grosse, A. Hartmann, A. Junghans, S. Mallion, G. Rusev, K. Schilling, W. Schulze et al., Nuclear Instruments and Methods in Physics Research Section A: Accelerators, Spectrometers, Detectors and Associated Equipment 555, 211 (2005)

[22] R. Schwengner, G. Rusev, N. Benouaret, R. Beyer, M. Erhard, E. Grosse, A.R. Junghans, J. Klug, K. Kosev, L. Kostov et al., Phys. Rev. C 76, 034321 (2007)

[23] R. Schwengner, G. Rusev, N. Tsoneva, N. Benouaret, R. Beyer, M. Erhard, E. Grosse, A.R. Junghans, J. Klug, K. Kosev et al., Phys. Rev. C 78, 064314 (2008)

[24] N. Benouaret, R. Schwengner, G. Rusev, F. Dönau, R. Beyer, M. Erhard, E. Grosse, A.R. Junghans, K. Kosev, C. Nair et al., Phys. Rev. C 79, 014303 (2009)

[25] B.L. Berman, S.C. Fultz, Rev. Mod. Phys. 47, 713 (1975)

[26] Kneissl, H. U Pitz, A. Zilges, Prog. Part. Nucl. Phys. 37, 349 (1996)

[27] N. Pietralla, Z. Berant, V.N. Litvinenko, S. Hartman, F.F. Mikhailov, I.V. Pinayev, G. Swift, M.W. Ahmed, J.H. Kelley, S.O. Nelson et al., Phys. Rev. Lett. 88, 012502 (2001)

[28] R. Raut, A.P. Tonchev, G. Rusev, W. Tornow, C. Iliadis, M. Lugaro, J. Buntain, S. Goriely, J.H. Kelley, R. Schwengner et al., Phys. Rev. Lett. 111, 112501 (2013)

[29] B. Löher, V. Derya, T. Aumann, J. Beller, N. Cooper, M. Duchêne, J. Endres, E. Fiori, J. Isaak, J. Kelley et al., Nuclear Instruments and Methods in Physics Research Section A: Accelerators, Spectrometers, Detectors and Associated Equipment 723, 136 (2013) 
[30] A. Sauerwein, K. Sonnabend, M. Fritzsche, J. Glorius, E. Kwan, N. Pietralla, C. Romig, G. Rusev, D. Savran, L. Schnorrenberger et al., Phys. Rev. C 89, 035803 (2014)

[31] R.S. Ilieva, P. Humby, N. Cooper et al. (2015), submitted

[32] V. Werner, Dipole strength distributions from HIGS experiments, in Fifteenth International Symposium on Capture Gamma-Ray Spectroscopy and Related Topics (CGS15) (2015)

[33] A. Spyrou, S.N. Liddick, A.C. Larsen, M. Guttormsen, K. Cooper, A.C. Dombos, D.J. Morrissey, F. Naqvi, G. Perdikakis, S.J. Quinn et al., Phys. Rev. Lett. 113, 232502 (2014)

[34] M. Guttormsen, T. Ramsøy, J. Rekstad, Nuclear Instruments and Methods in Physics Research Section A: Accelerators, Spectrometers, Detectors and Associated Equipment 255, 518 (1987)

[35] A. Schiller, L. Bergholt, M. Guttormsen, E. Melby, J. Rekstad, S. Siem, Nuclear Instruments and Methods in Physics Research Section A: Accelerators, Spectrometers, Detectors and Associated Equipment 447, 498 (2000)

[36] A.C. Larsen, M. Guttormsen, M. Krtička, E. Běták, A. Bürger, A. Görgen, H.T. Nyhus, J. Rekstad, A. Schiller, S. Siem et al., Phys. Rev. C 83, 034315 (2011)

[37] J.L. Tain, E. Valencia, A. Algora, J. Agramunt, B. Rubio, S. Rice, W. Gelletly, P. Regan, A.A. Zakari-Issoufou, M. Fallot et al., Phys. Rev. Lett. 115, 062502 (2015) 